\title{
Ischémie cellulaire et insuffisance rénale aiguë
}

La reperfusion d'un rein ischémique constitue la période de tous les dangers : l'accumulation $\mathrm{du}^{\mathrm{Ca}^{++}}$intracellulaire, la correction de l'acidose et, de ce fait, l'augmentation de l'activité d'enzymes, oxydases ou phospholipases susceptibles d'altérer les membranes biologiques, vont souvent aboutir à la mort cellulaire. Les inhibiteurs calciques, s'opposant à l'accumulation $\mathrm{du} \mathrm{Ca}^{++}$dans la cellule ischémiée pourraient constituer un utile traitement préventif des insuffisances rénales aiguës post-ischémiques.

\section{Michel Burnier}

Chef de clinique adjoint de néphrologie.

\section{Robert W. Schrier}

Professeur de médecine et chef de la division de néphrologie.

\section{ADRESSE ET TIRÉS À PART}

M. Burnier : division de néphrologie, centre hospitalier universitaire Vaudois, 1011 Lausanne, Suisse.

R.W. Schrier : departement of medicine, university of Colorado, school of medicine, Denver, CO, USA.

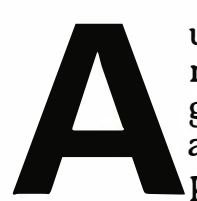

u cours des vingt dernières années, des progrès importants ont été accomplis dans la compréhension de la pathogénie, de la prévention, et du traitement de l'insuffisance rénale aiguë (IRA). L'amélioration des méthodes de réanimation et de nutrition et le développement des techniques de dialyse ont permis non seulement de réduire l'incidence de l'insuffisance rénale mais également d'en diminuer les complications. Cependant, malgré ces progrès, l'IRA demeure fréquente surtout chez les patients hospitalisés, probablement en raison de la survie prolongée de patients plus gravement atteints. En effet, les résultats récents d'une étude prospective ont montré que $4,9 \%$ d'un collectif de 2216 malades admis dans un milieu hospitalier développent un certain degré d'IRA au cours de leur séjour [1]. Dans certaines situations particulières, telles que les soins intensifs ou les suites d'une intervention cardiovasculaire, le pourcentage de patients développant une IRA peut même s'élever jusqu'à $50 \%$ [2]. Dans ces cas, la mortalité peut être importante puisqu'elle varie de 10 à $100 \%$ suivant que l'IRA est isolée ou associée à la dysfonction d'un ou de plusieurs autres organes [3]. Ainsi, pour diminuer la morbidité et la mortalité liées à l'IRA, il est important que des mesures préventives efficaces soient mises en œuvre lorsque la survenue d'une IRA est prévisible. Une telle prévention nécessite la compréhension des événements pathogéniques qui déclenchent et entretiennent le trouble de la fonction rénale. L'étude des anomalies cellulaires qui apparaissent au cours de l'IRA a ouvert de nouvelles perspectives dans le domaine de la prévention et nous nous proposons de les exposer dans les lignes qui suivent.

\section{Pathogénie de I'IRA: théories classiques}

L'IRA est caractérisée par une diminution du débit de filtration glomérulaire (FG) et par l'apparition d'une dysfonction tubulaire. 


\section{RÉFÉRENCES}

1. Hou SH, Bushinski A, Wish JB, Cohen J Harrington JT. Hospital-acquired renal insufficiency : A prospective study. Am $J$ Med $1983 ; 74: 243-8$.

2. Anderson RJ, Gross PA. Acute renal failure and toxic nephropathy. In : Klahr $S$, Massry SG, eds. Contemporary Nephrology. New York: Plenum, $1981 ; 1$ : 443-76.

3. Cameron JS. Acute renal failure in the intensive care unit today. Intensive Care Med $1986 ; 12: 64-70$.

4. Paller MS, Anderson RJ. Use of vasoactive agents in the therapy of acute renal failure. In : Brenner BM, Lazanus JM, eds. Acute Renal Failure. Philadelphia : WB Saunders, 1983 ; 28 : 723-40.

5. Cox JW, Baehler RW, Sharma H, et al. Studies on the mechanism of oliguria in a model of unilateral acute renal failure. $J$ Clin Invest 1974 ; 53 : 1546-58.

6. Venkatachalam MA, Bernard DB, Donohoe JF, Levinski NG. Ischemic damage and repair in the rat proximal tubule : differences among the S1, S2 and S3 segments. Kidngy Int $1978 ; 14: 31.49$.

7. Burke TJ, Cronin RE, Duchin KL, Peterson LN, Schrier RW. Ischemia and tubule obstruction during acute renal failure in dogs : Mannitol in protection. Am $J$ Physiol 1980 ; 238 : F305-14.

8. Stein JH, Gottschalk J, Osgood RW, Ferris T. Pathophysiology of a nephrotoxic model of acute renal failure. Kidney Int $1975 ; 8$ : 27-41.

9. Myers BD, Carrie BJ, Yee RR, Hilberman M, Michaels AB. Pathophysiology of hemodynamically mediated acute renal failure in man. Kidngy Int 1980 ; 18 : 495-504.

10. Siegel NJ. Amino acids and adenine nucleotides in acute renal failure. In : Brenner BM, Lazanus JM, eds. Acute Renal Failure. Philadelphia : Saunders, 1983 : 741-52.

11. Mason J, Beck F, Dorge A, Rick R, Thurau $K$. Intracellular electrolyte composition following renal ischemia. Kidney Int 1981; 20 : 61-70.

12. Humes HD. Role of calcium in pathogenesis of acute renal failure. Am J Physiol 1986 ; 250 : F579-89.
Plusieurs théories ont été proposées pour expliquer la baisse de la FG, les unes considérant avant tout des événements vasculaires et les autres insistant sur des altérations tubulaires. Les théories vasculaires impliquent soit une vasoconstriction rénale [4] qui entraîne une baisse du débit sanguin rénal, soit des altérations de la perméabilité glomérulaire [5]. S'il existe de nombreux arguments suggérant la participation de facteurs vasculaires au développement de l'IRA toxique ou ischémique, ces événements ne semblent toutefois déterminants que dans la phase initiale de l'IRA. En effet, l'augmentation du flux plasmatique rénal par des vasodilatateurs n'entraîne pas la normalisation de la fonction rénale lorsque la correction des anomalies vasculaires est tardive. Cette observation suggère la responsabilité d'autres facteurs dans le maintien de l'IRA. L'apparition d'altérations tubulaires constitue certainement la composante essentielle qui rend compte du maintien de la baisse de la FG au stade tardif de l'IRA. Les facteurs tubulaires qui peuvent être incriminés sont : (a) l'obstruction de la lumière tubulaire et (b) la réabsorption passive du filtrat glomérulaire. L'obstruction tubulaire a été mise en évidence dans plusieurs modèles d'IRA par des études morphologiques [6] et par des mesures de pression intratubulaire lors d'études de microponction [7]. Enfin, lorsque l'intégrité de l'épithélium tubulaire est atteinte, une réabsorption importante du filtrat glomérulaire à travers la paroi tubulaire a pu être démontrée dans un premier temps par des études de microinjection d'inuline marquée [8] et plus récemment par des méthodes de clearance fractionnelle de dextran/inuline [9]. Les molécules de dextran ayant un poids moléculaire supérieur à celui de l'inuline, un rapport de la clearance du dextran/clearance de l'inuline supérieur à 1 suggère la réabsorption d'inuline par les tubules.

L'ensemble de ces expériences confirme donc que des facteurs vasculaires et tubulaires partici- pent à la genèse de l'IRA, expérimentale ou clinique, et qu'aucun de ces éléments n'est capable à lui seul de rendre compte de la réduction considérable et persistante de la FG. Ces études physiopathologiques nous apprennent que la contribution relative de chacun de ces facteurs dépend avant tout de la cause de l'IRA et que les mécanismes responsables de l'installation de celle-ci different de ceux qui contribuent à son maintien.

\section{Aspects cellulaires de la pathogénie de I'IRA}

L'étude des altérations cellulaires qui se développent lors d'IRA a permis de mieux comprendre les différents événements vasculaires et tubulaires décrits précédemment. Malgré les difficultés présentées par cette approche, difficultés liées surtout à la complexité morphologique du rein et à la susceptibilité variable des différents segments tubulaires à l'ischémie ou aux toxiques, de rapides progrès ont été effectués ces dernières années, en particulier dans le domaine de l'ischémie rénale. C'est pourquoi les données présentées dans cet article concerneront surtout l'IRA ischémique. Deux phases sont à considérer dans le développement de la lésion cellulaire ischémique : la première se déroule pendant l'ischémie et la seconde lors de la reperfusion. (a) Lésions cellulaires apparaissant pendant l'ischémie. L'installation subite d'une ischémie cellulaire entraîne une cascade d'événements dont les plus critiques sont sans aucun doute la chute des taux d'adénosine triphosphate (ATP) et le développement du métabolisme anaérobie. Les concentrations d'ATP s'effondrent dans les secondes qui suivent l'apparition de l'ischémie [10]. En l'absence d'ATP, l'activité des pompes membranaires est interrompue et l'homéostasie électrolytique de la cellule ne peut être maintenue. Ainsi, Mason et al. ont montré qu'au cours d'une ischémie rénale, les concentrations intracellulaires de sodium et de 
chlore augmentent alors que celles de potassium, de phosphate et de magnésium diminuent [11]. On assiste également à une élévation du $\mathrm{Ca}$ intracellulaire, ce qui peut représenter une étape critique dans l'établissement de la lésion cellulaire ischémique. En effet, le $\mathrm{Ca}$ cytosolique joue un rôle important dans de nombreuses réactions enzymatiques intracellulaires et la stimulation de certaines enzymes telles que les phospholipases peut rapidement altérer l'intégrité cellulaire. Si l'augmentaton $\mathrm{du} \mathrm{Ca}$ cellulaire a été démontrée aussi bien in vivo qu'in vitro lors de l'ischémie rénale [12], les circonstances qui conduisent à cette élévation ne sont pas encore expliquées car peu d'études cinétiques ont été effectuées en cours d'ischémie. L'augmentation du $\mathrm{Ca}$ cytosolique peut être la conséquence d'une diminution de l'efflux de $\mathrm{Ca}$, phénomène ATP-dépendant, d'une augmentation de l'influx de $\mathrm{Ca}$ dans la cellule ou encore d'une dysfonction des organelles intracellulaires. Des études faites en ischémie sur des cellules rénales isolées n'ont pas permis de mettre en évidence une diminution de l'efflux du $\mathrm{Ca}$ immédiatement après 60 minutes d'hypoxie [13]. Au contraire Snowdowne et al. ont démontré une élévation de l'efflux de $\mathrm{Ca}$ proportionnelle à l'augmentation du $\mathrm{Ca}$ intracellulaire [13]. Dans ce contexte, l'apparition d'une acidose extra et intracellulaire liée au métabolisme anaérobie peut être importante. En effet, des études récentes n'ont pas montré d'augmentation de l'influx de $\mathrm{Ca}$ dans des tubules proximaux isolés et maintenus en anoxie pendant 30 minutes en présence d'une acidose extracellulaire [14]. Dès lors, on peut penser que l'augmentation du $\mathrm{Ca}$ cytosolique pendant l'ischémie représenterait essentiellement une redistribution $\mathrm{du} \mathrm{Ca}$ intracellulaire à partir des sites de stockage tels que les mitochondries ou le réticulum endoplasmique. La mise en évidence d'altérations dans les échanges de $\mathrm{Ca}$ à travers les membranes de ces organelles lors d'ischémie rénale joue en

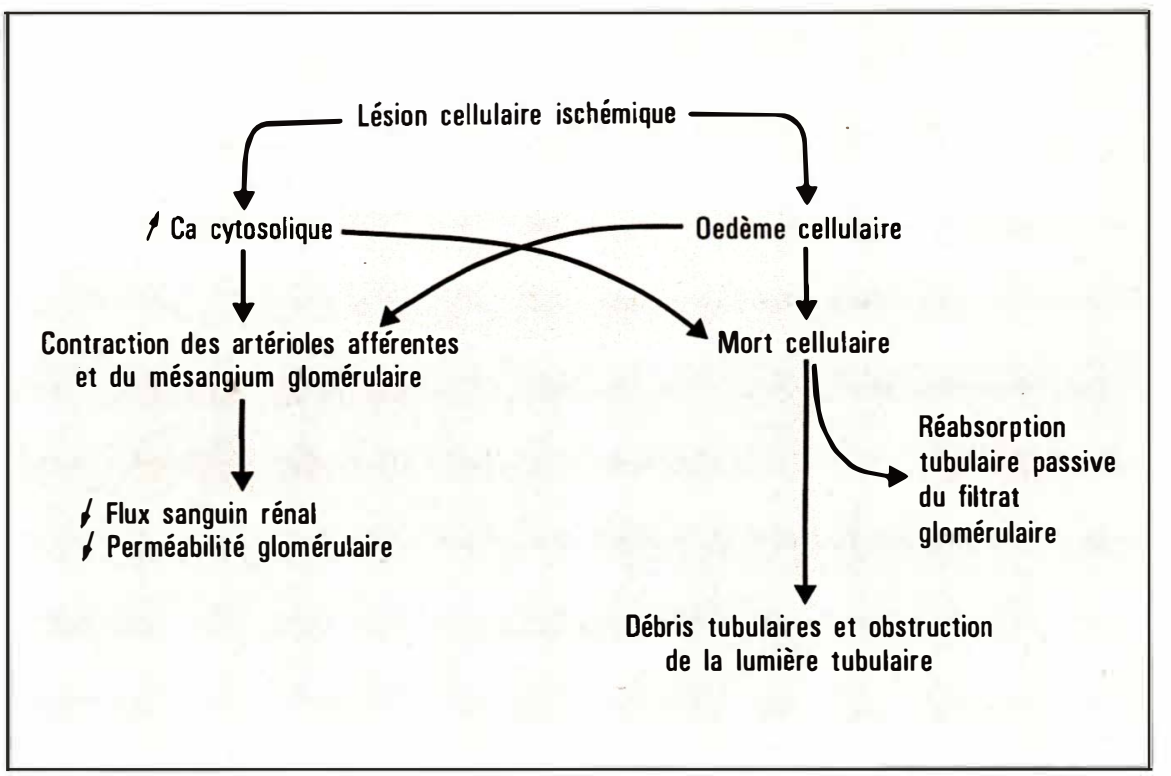

Figure 1. Schéma hypothótique des mécanismes impliqués dans la pathogénie de l'insuffisance rénale aiguë ischémique ou toxique.

faveur de cette hypothèse $[15,16]$. La conséquence principale de ces troubles de l'homéostasie cellulaire est le développement d'un œdème cellulaire dont les implications dans la pathogénie de l'IRA peuvent être multiples (figure 1). Tout d'abord, la tuméfaction des cellules endothéliales peut contribuer à la diminution de la perfusion rénale et prolonger la période d'ischémie. D'autre part; les troubles de la perméabilité membranaire peuvent être également à l'origine d'un œdème des cellules tubulaires qui favorisera la réabsorption passive du filtrat glomérulaire. Enfin si l'ischémie se prolonge, l'œdème cellulaire évoluera vers la nécrose avec pour conséquence l'obstruction de la lumière tubulaire par des débris cellulaires.

(b) Lésions cellulaires apparaissant au cours de la reperfusion. Lorsque la perfusion rénale est rétablie, les apports d'oxygène et de substrats métaboliques se normalisent rapidement et l'on s'attendrait à ce que de tels changements soient bénéfiques. En fait, de très nombreuses études effectuées dans divers organes (foie, cœur, reins) ont montré que la reperfusion aggravait parfois la lésion cellulaire ischémique. Trois facteurs principaux peuvent contribuer à ce phénomène : l'augmentation de l'apport d'oxygène, l'augmentation de l'apport en $\mathrm{Ca}$ et la correction de l'acidose cellulaire (figure 2, voir p. suivante).

- Oxygène. Dans les tissus sains, l'enzyme xanthine déshydrogénase permet de métaboliser la xanthine en acide urique en réduisant le NAD en NADH. Pendant l'ischémie, l'enzyme xanthine déshydrogénase est convertie en xanthine oxydase [17]. Il a été suggéré que l'augmentation du $\mathrm{Ca}$ intracellulaire active une protéase capable d'induire la formation de cette nouvelle enzyme. La xanthine oxydase utilise les molécules d'oxygène au lieu du NAD pour métaboliser la xanthine et ceci résulte en la formation de radicaux libres de l'oxygène. Cette conversion favorise donc la production de radicaux libres de l'oxygène en période de reperfusion lorsque l'apport en oxygène est suffisant. Ces radicaux libres entraîneront la peroxydation des acides gras libres et conduiront finalement à la dégradation des membranes cellulaires. Lors de l'ischémie rénale, Paller et al. n'ont en effet mis en évidence aucune augmentation des peroxydes lipidiques à la fin de 


\section{RÉFÉRENCES}

13. Snowdowne KW, Freudenrich CG, Borle AB. The effects of anoxia on cytosolic free calcium, calcium fluxes, and cellular ATP levels in cultured kidney cells. $J$ Biol Chem 1985 ; 260 : 11619-26.

14. Burnier M, Shanley P, Burke TJ, Schrier RW. Effect of extracellular acidosis on enhanced $\mathrm{Ca}$ influx in anoxic renal proximal tubules (PT). Kidney Int 1986 ; 29 : 299.

15. Amold PA, Lumlertgul D, Burke TJ, Schrier RW. In vitro versus in vivo mitochondrial calcium loading in ischemic acute renal failure. Am J Physiol 1985 ; 248 : F845-50.

16. Schieppati A, Wilson PD, Burke TJ Schrier RW. Effect of renal ischemia on cortical microsomal calcium accumulation. $A m J$ Physiol 1985 ; 249 : C476-83.

17. Mc Cord JM. Oxygen-derived free radicals in post-ischemic tissue injury. $N$ Engl $J$ Med 1985 ; 312 : 159-63.

18. Paller MS, Hoidal JR, Ferris TF. Oxygen-free radicals in ischemic acute renal failure in the rat. $J$ Clin Invest $1984 ; 74$ : 1156-64.

19. Arnold PE, Van Putten VJ, Lumlertgul D, Burke TJ, Schrier RW. Adenine nucleotide metabolism and mitochondrial $\mathrm{Ca}$ transport following renal ischemia. Am $J$ Physiol 1986 ; 250 : F357-67.

20. Wilson PD, Schrier RW. Nephron segment and calcium as determinants of ischemic cell death in primary renal cell cultures. Kidney Int $1986 ; 29: 1172-9$.

21. Schwertschlag U, Schrier RW, Wilson PD. Beneficial effects of calcium channel blockers and calmodulin binding drugs on in vitro renal cell anoxia. $J$ Pharmacol Exp Ther 1986 ; 238 : 119-24.

22. Weinberg JM. Oxygen deprivationinduced injury to isolated rabbit kidney tubules. J Clin Invest 1985; 76 : 1193-1208.

23. Brezis M, Rosen S, Silva P, Epstein FH. Renal ischemia : A new perspective. Kidng Int $1984 ; 26: 375-83$.

24. Simchowitz L. Intracellular $\mathrm{pH}$ modulates the generation of superoxyde radicals by human neutrophils. $J$ Clin Invest $1985 ; 76$ : 1079-89.

25. Patak RV, Fadem SZ, Lifschitz MD, Stein JH. Study of factors which modify the development of norepinephrine-induced acute renal failure in the dog. Kidney Int $1979 ; 15$ :

soixante minutes d'ischémie alors qu'après quinze minutes de réoxygénation une augmentation significative de la peroxydation des lipides est observée [18]. La protection cellulaire obtenue lors de l'administration d'inhibiteurs de la xanthine oxydase (allopurinol) ou d'agents qui neutralisent les radicaux libres (superoxyde dismutase, catalase) est un argument supplémentaire en faveur d'un rôle important des radicaux libres dans la pathogénie des lésions ischémiques.
- Calcium. La reperfusion des organes ischémiques s'accompagne également d'une augmentation de l'apport de $\mathrm{Ca}$ aux tissus, ce qui peut entraîner une élévation du $\mathrm{Ca}$ intracellulaire et finalement la destruction de la cellule par activation des phospholipases. Cette augmentation du $\mathrm{Ca}$ intracellulaire est due à plusieurs facteurs. Tout d'abord, l'altération des membranes cellulaires, qui apparaît en cours d'ischémie, augmente la perméabilité membranaire et permet au $\mathrm{Ca}$ extracellu-

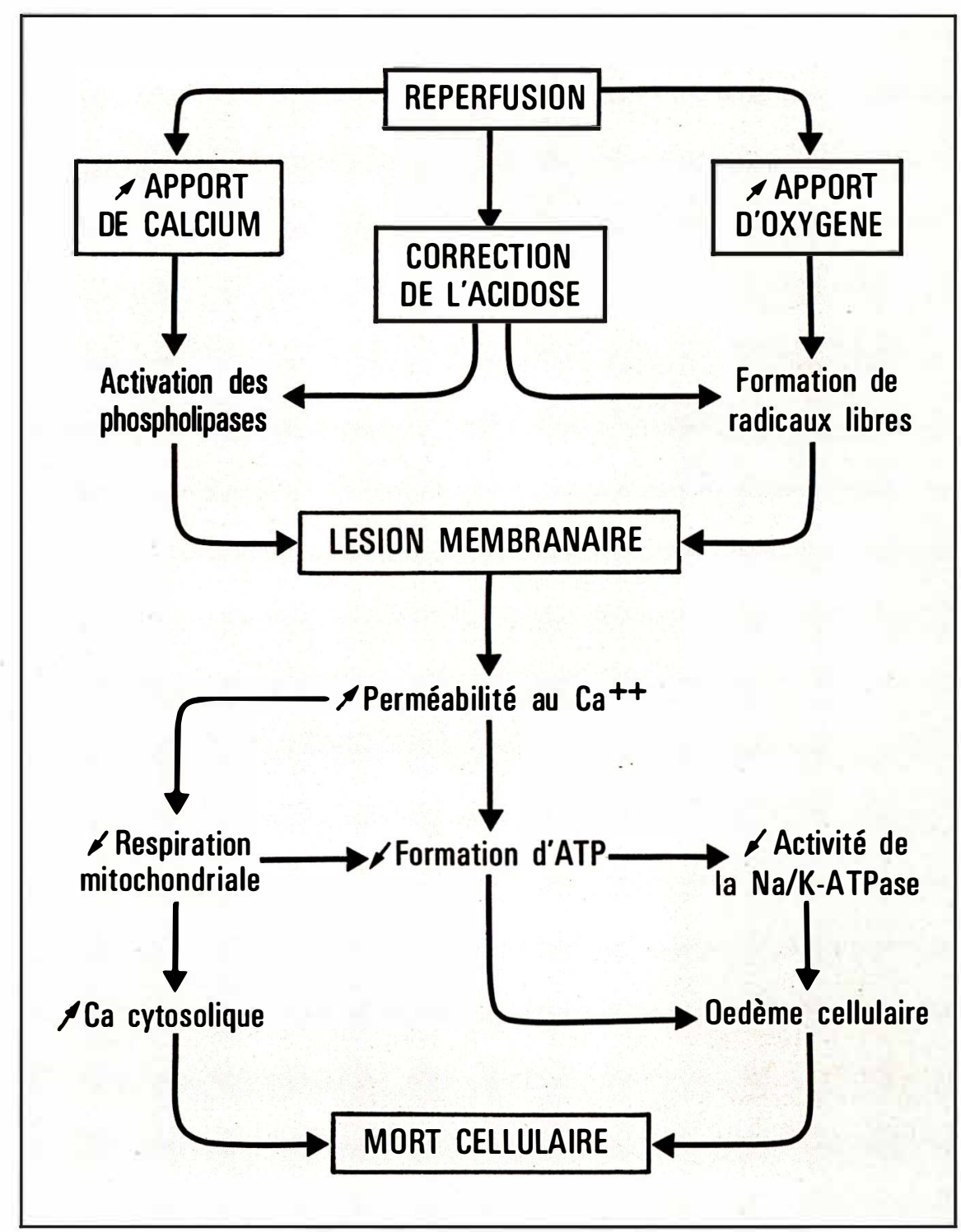

Figure 2. Représentation schématique des événements cellulaires qui peuvent se développer lors de la reperfusion d'un rein ischémique et qui peuvent entraîner la mort cellulaire. 
laire de pénétrer dans la cellule selon un gradient de concentration. D'autre part, la dysfonction des organelles intracellulaires (mitochondries, réticulum endoplasmique) perturbe les mécanismes intracellulaires responsables de l'homéostasie du $\mathrm{Ca}$ cytosolique. L'augmentation de l'influx de $\mathrm{Ca}$ dans la phase postanoxique a pu être démontrée in vitro sur des préparations de tubules isolés [14]. De plus, plusieurs études $d$ ischémie rénale faites chez le chien ont montré que l'activité des mitochondries, normale dans les premières heures de réoxygénation, diminue rapidement à partir de la troisième heure de reperfusion [15]. Dès ce moment, un déclin progressif de la respiration mitochondriale a été observé, correspondant à un accroissement du contenu en $\mathrm{Ca}$ des mitochondries. Simultanément, la capacité de ces mitochondries à contrôler l'augmentation du Ca extra-mitochondrial est fortement diminuée [19]. Ainsi, l'accumulation progressive de $\mathrm{Ca}$ par les mitochondries pendant la réoxygénation entraîne une "intoxication" des mitochondries et, secondairement, une élévation du $\mathrm{Ca}$, cytosolique.

L'étude de cellules rénales en culture lors d'ischémie a fourni d'autres éléments essentiels à la compréhension du rôle du $\mathrm{Ca}$ dans la lésion ischémique. En effet, les expériences de Wilson et al. ont montré que la survie de cellules rénales exposées à 45 minutes d'anoxie n'est pas prolongée en absence de $\mathrm{Ca}$ extracellulaire au cours de la réoxygénation [20]. Cependant, l'absence de $\mathrm{Ca}$ extracellulaire durant les deux premières heures de réoxygénation uniquement est associée à une prolongation significative de la survie des cellules (figure 3). De même, l'adjonction d'un inhibiteur du transport de $\mathrm{Ca}$ dans le milieu de culture permet d'augmenter la survie de cellules tubulaires anoxiques [21].

- Acidose. Le rôle de l'acidose dans le développement des lésions ischémiques est encore mal compris et les résultats expérimentaux

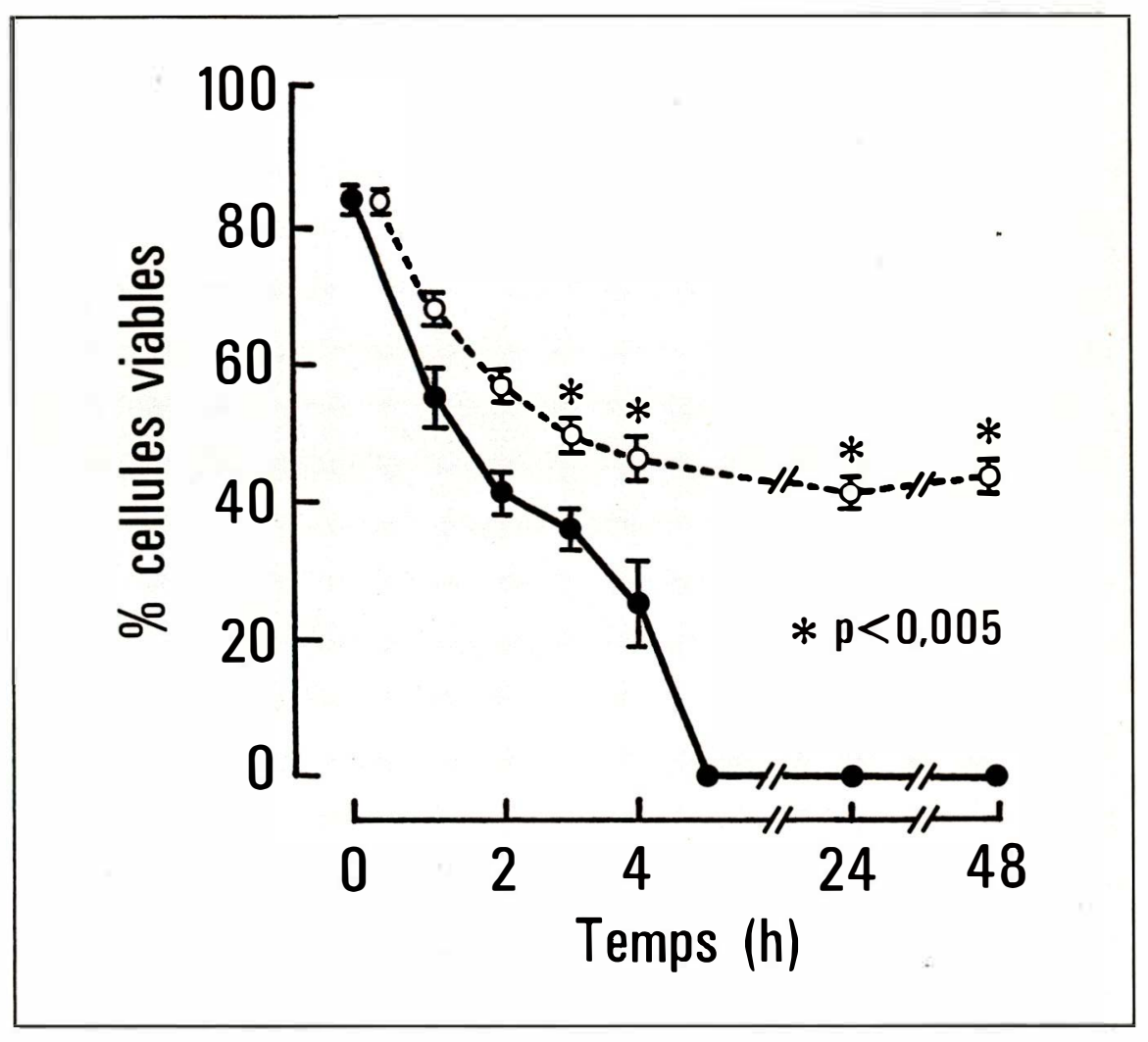

Figure 3. Effet de la concentration extracellulaire de Ca sur la survie de segments tubulaires proximaux $\mathrm{S} 3$ en culture, lors de la réoxygénation après 45 minutes $d^{\prime}$ anoxie. - segments incubés dans un milieu contenant 1 mM de Ca. O---O segments incubés dans un milieu sans $\mathrm{Ca}$ pendant les deux premières heures de réoxygénation puis avec $1 \mathrm{mM}$ de Ca. (Adapté de [20]).

sont contradictoires. Les études qui ont évalué l'effet de l'acidose sur la récupération fonctionnelle. $\mathrm{du}$ rein après ischémie, lors de la préservation d'organes par exemple, n'ont pas montré de bénéfice réel de l'acidose. Par contre, les études biochimiques de l'effet de l'acidose sur la lésion cellulaire ischémique suggèrent un effet protecteur [22]. Ces deux observations contradictoires à première vue, pourraient toutefois être réconciliées par le fait qu'en acidose, de nombreuses réactions enzymatiques sont inhibées. L'inhibition de ces processus enzymatiques pourrait prolonger la survie des cellules ischémiques en réduisant la demande énergétique ou en inhibant la dégradation des membranes cellulaires liée à l'activation des phospholipases. La diminution de l'activité métabolique compromet cependant l'activité basale des cellules et les différentes fonctions du rein ne peuvent être maintenues. Le rôle de l'activité métabolique cellulaire dans le développement des lésions ischémiques a été très bien étudié par Brezis et al. dans des préparations de reins isolés et perfusés [23]. Ces études ont montré que la distribution des lésions ischémiques du rein est liée à l'activité métabolique des différents segments tubulaires et que l'inhibition du transport tubulaire de certains segments entraîne une protection morphologique de ces derniers.

Ainsi, la correction de l'acidose lors de la reperfusion représente 


\section{RÉFÉRENCES}

26. Lindner A. Synergism of dopamine and furosemide in diuretic resistant, oliguric acute renal failure. Nephron $1983 ; 33$ : 121-6.

27. Anderson RJ, Linas SL, Berns AS, et al. Nonoliguric acute renal failure. $N$ Engl $J$ Med $1977 ; 296$ : 1134-8.

28. Burke TJ, Arnold PE, Gordon JA, Bulger RE, Dobyan DC, Schrier RW. Protective effect of intrarenal calcium membrane blockers before or after renal ischemia. $J$ Clin Invest $1984 ; 74: 1830-41$

29. Shapiro JI, Cheung C, Itabashi A, Chan L, Schrier RW. The effect of verapamil on renal function after warm and cold ischemia in the isolated perfused kidney. Transplantation 1985 ; 40 : 596-600.

30. Burnier $\mathbf{M}$, Van Putten V, Wilson $\mathbf{P}$, Burke TJ, Schrier RW. Beneficial effects of verapamil $(\mathrm{V})$ and nifedipine $(\mathrm{N})$ on $\mathrm{Ca}$ influx and cell viability in anoxic renal cortical proximal tubules (CPT). Miner Electrolyte Metab 1985 ; 11 : 390 (Abstract).

31. Wagner K, Albrecht S, Neumayer $\mathrm{HH}$. Prevention of delayed graft function in cadaveric kidney transplantation by a calcium antagonist. Preliminary results of two prospective randomized trials. Transplant Proc 1986; 18 : 510-5.

32. Duggan KA, MacDonald GJ, Charlesworth JA, Pussel BA. Verapamil prevents posttransplant oliguric renal failure. Clin Nephrol certainement un facteur déterminant dans la pathogénie de l'IRA car l'augmentation du $\mathrm{pH}$ intracellulaire entraîne une augmentation du métabolisme cellulaire et l'activation des phospholipases dont le $\mathrm{pH}$ optimal est élevé. De plus, nous avons pu démontrer récemment sur une préparation de tubules proximaux maintenus en anoxie pendant 30 minutes que la correction du $\mathrm{pH}$ extracellulaire est associée à un influx rapide de $\mathrm{Ca}$ à l'intérieur des cellules tubulaires [14]. Cette observation suggère que l'influx de $\mathrm{Ca}$ dans les cellules ischémiques a lieu essentiellement pendant la période de reperfusion lors de la correction de l'acidose. D'autre part, une étude récente sur des polynucléaires neutrophiles humains a démontré que la génération de radicaux superoxydes est dépendante du $\mathrm{pH}$ intracellulaire, ce qui pourrait représenter un autre mécanisme par lequel l'acidose influencerait l'évolution de la lésion cellulaire ischémique [24].

\section{Prévention}

Parce que l'oligurie est l'un des signes les plus apparents au cours de l'IRA, il n'est pas surprenant que les diurétiques aient été parmi les premières substances utilisées dans sa prévention. Actuellement, le furosémide et le mannitol sont employés couramment dans des situations cliniques où le risque de développer une IRA est élevé. Les mécanismes par lesquels ces substances préviennent l'IRA sont multiples. En effet, des études cliniques et expérimentales ont montré que les diurétiques peuvent : (a) augmenter le flux sanguin rénal et améliorer l'hémodynamique rénale, (b) induire une diurèse osmotique et diminuer l'obstruction tubulaire, (c) diminuer l'œdème cellulaire et (d) agir comme tampons des radicaux libres. Finalement, les études de Brezis et al. suggèrent que l'inhibition du transport tubulaire induit par les diurétiques participe également à leur effet protecteur [23].

La correction des anomalies hémodynamiques et en particulier la normalisation du flux sanguin rénal n'a pas rencontré un succès comparable à celui des diurétiques dans la prévention de l'IRA. Parmi les nombreux agents pharmacologiques testés dans diverses formes d'IRA expérimentales et apportant une protection dans certains modèles, ceux utilisés en clinique sont rares. Toutefois les travaux de Patak et al. ont montré que l'association d'un diurétique et d'un agent vasoactif est plus efficace que leur utilisation isolée [25]. Cette observation expérimentale a été confirmée récemment par quelques études cliniques non contrôlées qui ont montré que l'association dopaminefurosémide diminuait la durée de l'anurie lors d'IRA [26]. En outre, cette combinaison a permis de rétablir une diurèse, élément très important puisque la morbidité et la mortalité liées à l'IRA sont significativement réduites dans l'IRA non oligurique [27]. Parmi les substances qui agissent aussi bien au niveau vasculaire que tubulaire, les inhibiteurs du $\mathrm{Ca}$ sont probablement les plus prometteurs. Qu'il s'agisse du vérapamil, de la nifédipine ou du diltiazem, les inhibiteurs du calcium ont une propriété commune au niveau de la musculature lisse vasculaire ou du myocarde qui consiste à inhiber l'influx de calcium par les canaux lents des membranes cellulaires. Ainsi, dans des situations cliniques pathologiques telles que l'ischémie rénale, les inhibiteurs du $\mathrm{Ca}$ diminuent la vasoconstriction et augmentent le flux sanguin rénal. L'effet des inhibiteurs du calcium sur les cellules épithéliales est moins défini. Toutefois, ils pourraient diminuer le degré de nécrose tubulaire en prévenant l'accumulation de $\mathrm{Ca}$ dans les cellules tubulaires. L'effet protecteur des inhibiteurs du $\mathrm{Ca}$ a été démontré par plusieurs auteurs dans des modèles animaux d'IRA ischémique. Ainsi, après 30 minutes de clampage de l'artère rénale chez le chien, la récupération de la fonction rénale est significativement meilleure chez les animaux prétraités avec 
du vérapamil ou de la nifédipine [28]. Le vérapamil améliore aussi la fonction rénale au décours d'une ischémie chaude ou froide dans des modèles de reins isolés et perfusés [29].

Enfin, plusieurs travaux in vitro ont démontré que les inhibiteurs du Ca pouvaient prévenir le développement des lésions ischémiques indépendamment de tout effet vasculaire. Ainsi la préincubation avec du vérapamil de cellules tubulaires en culture a permis de diminuer leur mortalité après une exposition de 45 minutes à l'anoxie [21]. L'analyse de la cinétique du Ca sur une préparation de tubules proximaux exposés à l'anoxie pendant 30 minutes suggère que l'effet protecteur des inhibiteurs du $\mathrm{Ca}$ est dû à une diminution du $\mathrm{Ca}$ membranaire [30]. Ainsi, il paraît de plus en plus évident que les inhibiteurs du $\mathrm{Ca}$ ont un effet direct sur la lésion ischémique tubulaire indépendamment de tout effet vasculaire. Quelques études cliniques ont récemment confirmé que les inhibiteurs du $\mathrm{Ca}$ peuvent être utiles dans le traitement préventif de l'IRA [31, 32]. Ces études concernent essentiellement le domaine de la transplantation rénale, situation dans laquelle l'ischémie est prévisible et inévitable.

En conclusion, l'étude de la physiopathologie de l'insuffisance rénale au niveau cellulaire a débouché sur de nouvelles possibilités de la prévenir. D'autres alternatives thérapeutiques devraient aboutir dans un avenir assez proche car une littérature expérimentale abondante existe déjà sur les moyens de prévenir la formation de radicaux libres ou d'éviter la déplétion cellulaire en ATP. Il en va de même pour la prévention de l'IRA d'origine néphrotoxique, domaine dans lequel des progrès considérables sont en cours. La prévention efficace de l'insuffisance rénale ischémique ou toxique permettra ainsi de réduire la morbidité et la mortalité qui sont attachées à cette affection

\section{Summary}

Acute renal failure remains a major cause of morbidity and mortality, and the development of effective means to decrease its incidence is necessary. Advances in the understanding of the pathophysiology of acute renal failure often lead to new therapeutics modalities. During the past several years, the cellular abnormalities occurring during an ischemic or toxic renal injury have been examined in detail and the role of several events such as ATP depletion, calcium accumulation or the formation of free radicals, has been determined more precisely. These progresses have supplied important information to improve the pharmacologic prevention of acute renal failure. In particular, the potential beneficial effects of agents that interfere with calcium entry or calcium mediated damage have raised a considerable interest. 